\title{
Precision Control of Pulse Widths for Charge Balancing in Functional Electrical Stimulation
}

\author{
Yi-Kai Lo, Richard Hill, Kuanfu Chen, and Wentai Liu
}

\begin{abstract}
Maintaining a balance charge is one of the key factors to ensure a safe neural stimulation employing biphasic current stimulus. However, a zero net charge is difficult to achieve due to the fabrication variation of stimulation drivers. Moreover, even with a perfectly matched cathodic and anodic current stimulus, a non-zero residual charge is still built up due to the inter-pulse delay adopted in the stimulation pattern. During this period, the charge injected by the first stimulus pulse is leaked to the surrounding tissue and thus, the remaining charge cannot be completely removed by the following compensating current stimulus even with matched intensity. In this paper, we present a charge-balancing scheme by precisely controlling the pulse width of current stimulus. Charge balance is achieved by using the residual voltage as a feedback signal to control the pulse width of next stimulus. Simulated in Matlab/Simulink, the proposed scheme is shown to mitigate up to $15 \%$ intensity mismatch of biphasic current pulses with $1 \mathrm{~ms}$ inter-pulse delay. For a stimulus with constantly varying intensity, such as retinal and cochlear implants, the proposed scheme is also capable of maintaining a balance charge for a safe neural stimulation.
\end{abstract}

Index Terms-Stimulator, biphasic stimulation, functional electrical stimulation (FES), charge balance, retinal implant, neural implant, cochlear implant, neural damage,

\section{INTRODUCTION}

Functional electrical stimulation (FES) has been used in biomedical implants to restore lost functions in individuals by delivering balanced electrical charge to evoke action potentials in biological tissue. [1] FES typically delivers biphasic current to depolarize the neural membrane, while aiming to maintain a zero Faradic charge residual at the stimulation site. When a non-zero residual charge is established at stimulation site, a DC current flows into the surrounding tissue, generally thought to cause neural damage as a result of the excessive generation of toxic byproducts from electrochemical reactions, $\mathrm{pH}$ changes, gas formation, and electrode dissolution [2]. Consequently, significant research endeavors have been devoted to ensure safe electrical stimulation [3]-[7].

A simple passive method to avoid DC current is the insertion of DC blocking capacitor between each stimulating electrode and stimulator output. However, the value of the necessitated capacitance is usually large, impeding the use of an on-chip capacitor [3]. An alternative is to use discrete capacitors, but this approach inevitably increases the footprint of implantable devices, especially for a size-limited

Yi-Kai Lo, Richard Hill, Kuanfu Chen, and Wentai Liu are with the Bioengineering Department, University of California, Los Angeles, CA90095 USA, (e-mail:yikai.lo@ucla.edu). high-density neural implant with hundreds of stimulation outputs, e.g. retina prosthesis. Shorting the stimulating electrode to the circuit ground or the reference electrode after each stimulation cycle has also been widely used to mitigate the residual charge [4]-[5]. The shorting operation provides a discharge path for the residual charge, but its effectiveness largely depends on the electrode impedance (electrode time constant), the amount of residual charge, and the time period between each stimulating pulse (time available for discharge). Insufficient discharge time leads to the build-up of residual voltage at the electrode site. Moreover, in a multiple-channel stimulator, if one channel is stimulating while an adjacent channel is shorting to circuit ground, the majority of the stimulating electrical charge might not penetrate the tissue and return via the far-away reference electrode, as intended. Instead, charge can flow superficially to the adjacent shorting electrode. Thus, the effectiveness of stimulation is reduced. Lastly, passive charge-control techniques usually do not measure the electrode potential before or after stimulation to guarantee no neural damage can occur.

Active balancing schemes have been reported in [6],[7]. Predefined current pulses at the end of each stimulation cycles are used to remove residual charge. Nonetheless, the effect of the balancing short pulses has not yet been investigated in clinical animal or human studies. There is a possibility that the inserted short pulses might result in unwanted neural responses. Applying an offset DC current into the electrode has also been used to cancel the mismatch in a biphasic current stimulus with fixed intensities. However, this technique has not yet been shown to be experimentally effective for current stimulus with changing intensities.

The main cause of non-zero residual charge is believed to be the mismatch between the cathodic and anodic current stimulus. It has been demonstrated that a current mismatch of $>100 \mathrm{nA}$ is highly correlated to neural damages [3]. However a perfect charge balance of current stimulus is difficult to achieve as a result of the intrinsic mismatch of current sink and source drivers caused by semiconductor process variations ( 1-2\%). Interestingly, even with a perfect matched cathodic and anodic current pulse, zero residual charge is still not achievable due to the inter-pulse delay typical adopted in modern biphasic stimulation. A phenomenon we have observed is that the Faradic charge transfer resistance in the electrode model creates a leakage charge during the period of inter-pulse delay. Thus, previous works focusing on reducing imbalance between cathodic and anodic stimulation current pulses in hopes of reducing residual charges might not be effective ways to ensure a safe neural stimulation [3],[4], [8]. 


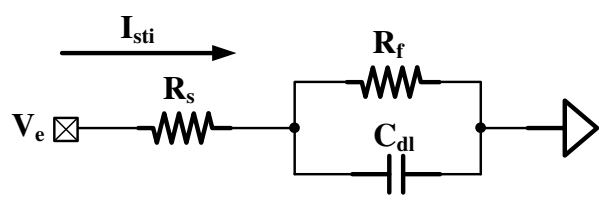

Fig. 1. Simplified electrode-electrolyte interface mode

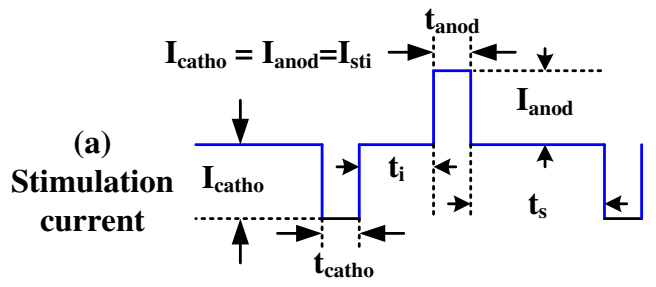

(b)

Voltage on

electrode

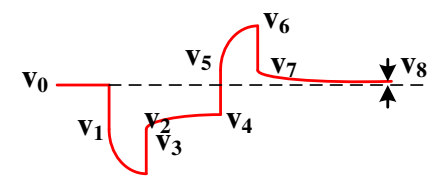

Fig. 2. Waveforms of balanced stimulation current and corresponding electrode voltage using the electrode model in Fig.1.

In this paper, we propose an active charge-balancing scheme to effectively remove residual charge even when an inter-phase delay is inserted into the stimulation pattern. Instead of spending significant efforts to match the biphasic current amplitudes and designing sophisticated charge compensating circuits, a precise control of the width of either the anodic or the cathodic current pulses is used for charge balancing. No DC blocking capacitors or additional compensating pulses is required. The proposed approach has been shown effective both in theoretical derivation and simulation with Matlab/SimuLink, and can be embedded in the design of digital controller of neural stimulators.

The remainder of this paper is organized as follows. Section II provides a brief introduction to residual charge formation, while Section III presents the proposed chargebalancing scheme. Section IV and V, respectively, presents the simulation results and conclusion.

\section{Residual Charge Formation}

A simple electrode-electrolyte model is used in the paper, as shown in Fig. 1. Assuming the impedance of the counter/return electrode can be neglected. $\mathrm{R}_{\mathrm{S}}$ is the tissue spreading resistance. $\mathrm{C}_{\mathrm{dl}}$ is the double layer capacitance forming at the electrode-electrolyte interface and $R_{f}$ is the Faradic charge transfer resistance. Warburg impedance is not included in this model. $\mathrm{V}_{\mathrm{e}}$ is the residual voltage measured between stimulating electrode and reference/return electrode.

There are several stimulus parameters in biphasic current mode stimulation: intensity and width of cathodic and anodic current pulses, the duration of inter-pulse delay, and the sequence of stimulus polarities. A cathodic first current pulse followed by an anodic current pulse for charge balancing is usually adopted in current mode stimulation and is used in our analysis. When the widths of both cathodic and anodic pulses are the same with no inter-pulse delay, the residual charge is simply due to the mismatch between the stimulus intensities. However, an inter-pulse delay is usually adopted to lower the threshold of the neurons [2]. During this period, the charge stored in $\mathrm{C}_{\mathrm{dl}}$ is leaked out through $\mathrm{R}_{\mathrm{f}} / \mathrm{R}_{\mathrm{s}}$. A bi-phasic current stimulation waveform is illustrated in Fig. 2(a). $I_{\text {catho }}$ and $I_{\text {anod }}$ are the intensity of cathodic and anodic current, respectively, while $t_{\text {catho }}$ and $t_{\text {anod }}$ are the width of the corresponding current pulses. $t_{i}$ is the inter-pulse delay and $t_{s}$ is the time before the starting of next stimulus. Fig. 2(b) shows the corresponding voltage waveform on the electrode. $V_{1}$ equals the voltage drop on Rs due to the starting transient increase of cathodic current. $\mathrm{I}_{\text {sti }}$ then charges $C_{d l}$ and $R_{f}$, leading to $V_{2} . V_{3}$ is one $V_{1}$ higher than $V_{2}$ since the current stimulus goes to zero. During the inter-pulse delay, $V_{3}$ increase to $V_{4}$ as a result of the leakage charge, where

$V_{4}=V_{3} * e^{\frac{-t_{i}}{R_{f} C_{d l}}}=\frac{I_{\text {stim }}}{C_{d l}}\left(1-e^{\frac{-t_{\text {catho }}}{R_{f} C_{d l}}}\right) * e^{\frac{-t_{i}}{R_{f} C_{d l}}}$

A compensating anodic pulse is then applied, resulting in $\mathrm{V}_{5,6,7}$. After the anodic current pulse, $\mathrm{V}_{8}$ can be expressed as

$V_{8}=V_{7} * e^{\frac{-t_{s}}{R_{f} C_{d l}}}$
$=\frac{I_{s i t}}{C_{d l}}\left(\left(1-e^{\frac{-t_{\text {canho }}}{R_{f} C_{d l}}}\right) * e^{\frac{-t_{i}}{R_{f} c_{d l}}}-\left(1-e^{\frac{-t_{\text {anod }}}{R_{p} C_{d l}}}\right)\right) * e^{\frac{-t_{s}}{R_{f} C_{d l}}}$

It can be seen from Eq. (2) a residual voltage on the electrode exists when $t_{i}$ is not zero with matched biphasic current pulses. $t_{s}$ should thus be sufficiently long to discharge the residual charge, otherwise this residual electrode voltage can accumulate and increase with every stimulation cycle. This problem can also be intensified by the amplitude mismatch of the current sink and source drivers. Without correction, the electrode might reach dangerous voltage levels that can lead to corrosion and generation of toxicity in the surrounding tissues and create a DC current flowing into surrounding tissue.

\section{Active Charge Balancing BASEd ON PRECISION PULSE WidTH CONTROL}

Fig. 3 presents the system diagram of the proposed charge balancing scheme. Electrode voltage is examined by turning on/off the switch $\phi_{1}$ after each stimulus. The feedback sensor monitors the electrode voltage to send the information to the proportional-integral-derivative (PID) controller. In the circuit implementation, this feedback sensor could be a multi-bit ADC or a simple comparator. If a positive residual voltage is observed, the width of cathodic current is increased or width of anodic current is decreased. The same principle of operation applies when a negative residual voltage is monitored. A modified Ziegler-Nichols rule-based tuning is used in the PID controller. The parameter of $\mathrm{K}_{\mathrm{P}}, \mathrm{K}_{\mathrm{I}}$, and $\mathrm{K}_{\mathrm{D}}$ are set as $\mathrm{K}_{\mathrm{P}}=0.6 \mathrm{~K}_{\mathrm{U}}, \mathrm{K}_{\mathrm{I}}=2 \mathrm{~K}_{\mathrm{P}} / \mathrm{T}_{\mathrm{U}}$, and $\mathrm{K}_{\mathrm{D}}=\mathrm{K}_{\mathrm{P}} * \mathrm{~T}_{\mathrm{U}} / 8$, where $\mathrm{T}_{\mathrm{U}}$ is 0.12 and $K_{U}$ is $6 e-4$ to ensure there is no overshooting [9]. However, in a case where more information is known about the system, different PID coefficients can be chosen to minimize the settling time. Simulation parameters were based on the retinal prosthesis chip developed in our laboratory [5]. 
With a $2 \mathrm{MHz}$ system clock for the controller circuit in the chip to command the stimulation drivers, 500ns can be

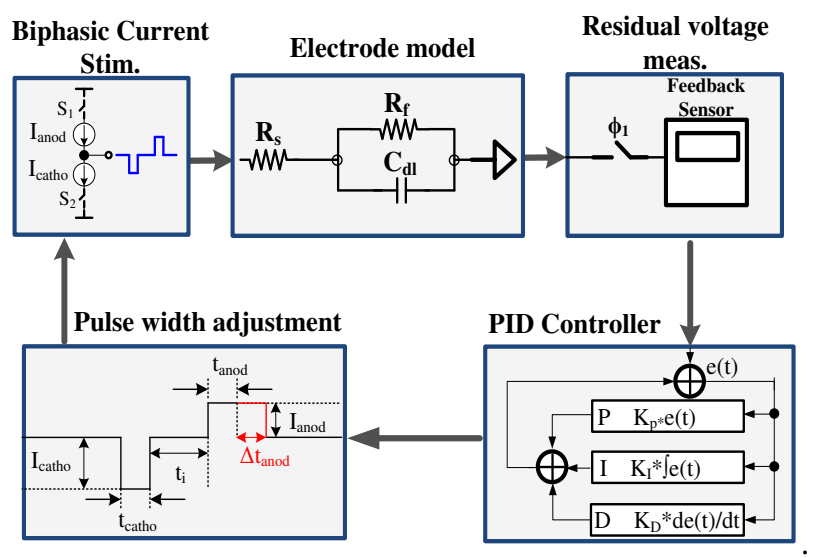

Fig. 3. System diagram of charge balancing scheme

current of $3 \mu \mathrm{A}$, the compensation charge delivered can be any integer multiple of $1.5 \mathrm{pC}$. This is smaller and more precise than $1.2 \mathrm{nC}$ reported in [6].

\section{Simulation RESUlts}

Matlab/Simulink is used to implement and simulate the proposed charge balancing scheme. The electrode model is configured as the following parameters: $\mathrm{R}_{\mathrm{f}}=1 \mathrm{M} \Omega, \mathrm{C}_{\mathrm{dl}}=10 \mu \mathrm{F}$, and $R_{s}=10 \mathrm{k} \Omega$ [4]. Only the width of the anodic pulse was adjusted to balance the charge because for most applications, the leading phase is the main stimulation pulse and the following phase is the compensation pulse. To ensure that enough charge is delivered in the stimulation pulse, it follows that only the compensation pulse should be adjusted. It is also notable that adjusting the width of the cathodic phase or both phases are also viable solutions. The electrode was stimulated with $500-\mathrm{Hz}$ cathodic first biphasic constant current pulses, as shown in Fig. 2(a). The $t_{i}$ is $1 \mathrm{~ms}$, $t_{\text {catho }}$ was $1 \mathrm{~ms}$, and $t_{\text {anod }}$ was adjustable between 0.7 and $1.4 \mathrm{~ms}$ (but started at $1 \mathrm{~ms}$ ). $\mathrm{t}_{\mathrm{s}}$, is adjusted so that each stimulation cycle is $20 \mathrm{~ms}$.

The simulation is performed with two types of base amplitude scenarios. First, they were tested with a fixed base current stimulus of $200 \mu \mathrm{A}$. Second, they were tested with variable base amplitudes between 0-200 $\mu \mathrm{A}$. For each stimulation cycle, the greatest electrode voltage during the post-stimulation period was recorded, corresponding to the point $\mathrm{V}_{7}$ in Fig. 2(b).

The system is verified to properly balance charge under a constant amplitude mismatch related to fabrication variation of the stimulation drivers. A 4-bit ADC is used to monitor the residual electrode voltage. The material of the electrode is assumed to be platinum which is commonly used in retinal prosthesis [10]. The water window of platinum electrode is thus set as between $-0.6 \mathrm{~V}$ and $0.8 \mathrm{~V}$ for a safe electrical operation boundary. Fig. 4(a) shows the $V_{7}$ for 20 cycles without any feedback control. The electrode voltage exceeds the water window for the cases of Cathodic $-15 \%$ and Cathodic $+15 \%$. Furthermore, even though the cases of Cathodic $-5 \%$ and Cathodic $+5 \%$ do not exceed the water window, a DC current would also be created and flow into tissue. Fig. 4(b) shows residual electrode voltage after using

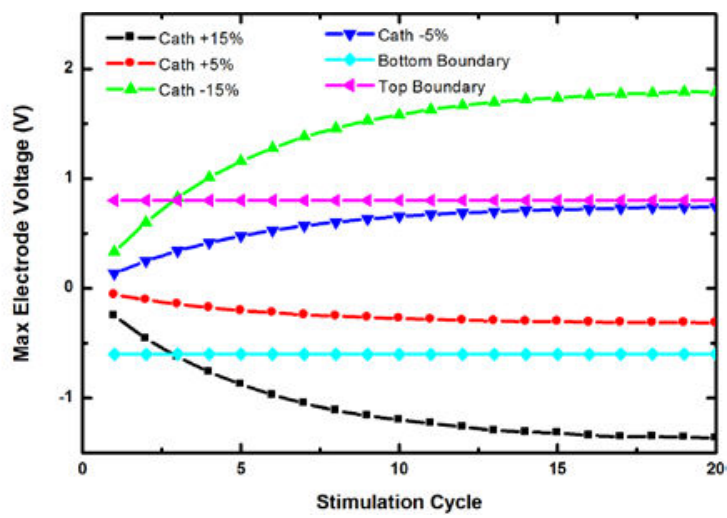

(a)

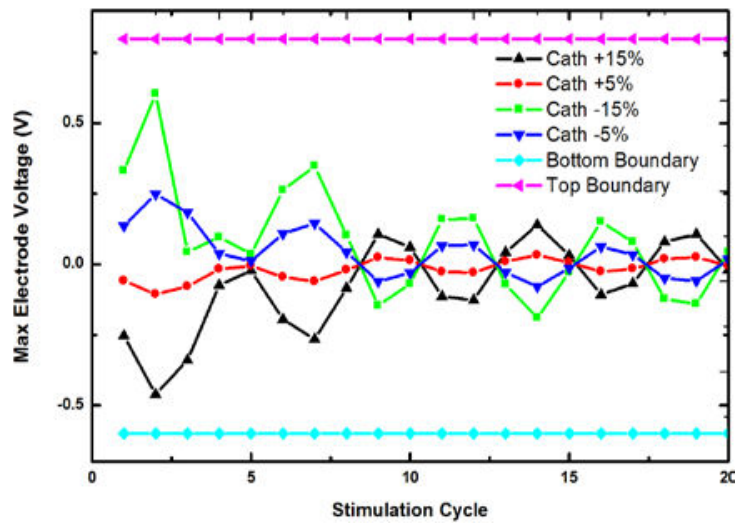

(b)

Fig. 4. The max electrode voltage during post-stimulation time (a) without control (b) with proposed control scheme for a 4-bit ADC and constant stimulation amplitudes.

the proposed charge balancing scheme. The electrode voltage approaches $0 \mathrm{~V}$ and gradually converges to this value while slightly oscillating around it.

The intensity of the current stimulus in an actual implant would be changed constantly depending on the subject's environment. For example, for a patient with a retinal or cochlear implant, the amplitude of the stimulation will vary according to the image/audio perceived. A simulation is therefore performed with varying current intensities but a fixed ratio of current mismatch. Fig. 5(a) shows the electrode voltage without charge balancing and current stimulus with randomly varying intensity of $0-200 \mu \mathrm{A}$ is applied. As predicted, the electrode voltage reaches potentially damaging levels. It can be also noted that the electrode voltage in Fig. 5(a) does not reach plateau because the stimulus has varying current intensities. Fig. 5(b) shows a proper balancing of the mismatch charge with the proposed scheme. In the first 20 cycles, the electrode voltage approaches $0 \mathrm{~V}$. In this scenario, the pulse width is constantly being adjusted and is a continuous background operation. For a cathodic $+15 \%$ current mismatch, the anodic pulse width is adjusted between $1.001 \mathrm{~ms}$ to $1.217 \mathrm{~ms}$. The charge-balancing scheme is effective though the electrode voltage is continually oscillating around $0 \mathrm{~V}$ and never crosses the water window. Lastly, a comparator (1-bit ADC) is used to replace the 4-bit ADC to repeat previous simulation. Fig. 6 shows the residual electrode voltage after 20 pulse cycles. The amplitudes of the 


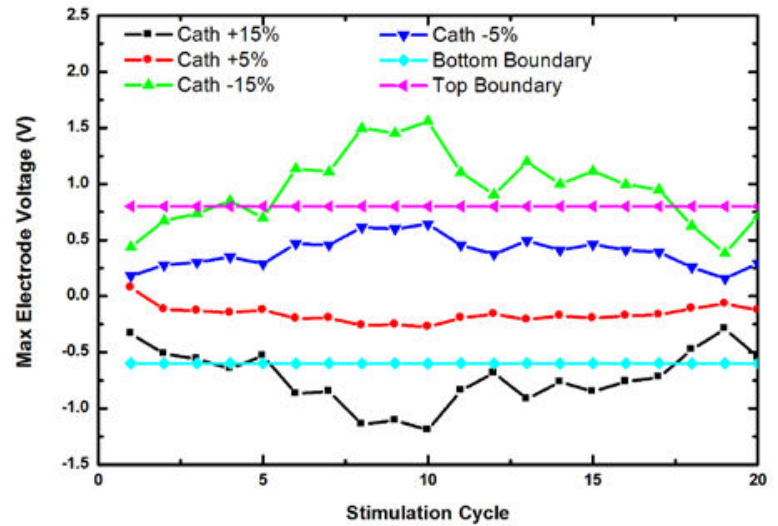

(a)

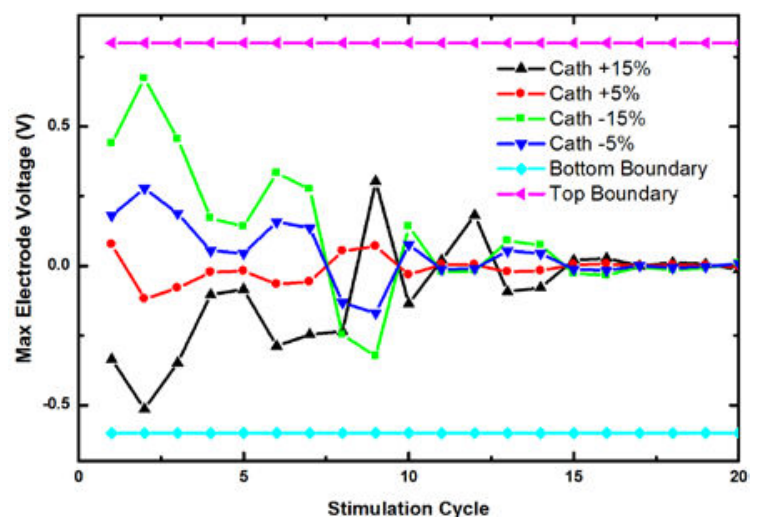

(b)

Fig. 5. The max electrode voltage during post-stimulation time (a) without control (b) with proposed control scheme for a 4-bit ADC and variable stimulation amplitudes

oscillations are noticeably larger, but the charge balancing system still keeps the electrode voltage within safe ranges. Since comparator can only provide 1-bit information to the PID controller, it is challenging to achieve a fast settling of electrode voltage. Thus, in the hardware implementation, trade-offs must be made to decide an optimal resolution of ADC in terms of power, area, and settling speed.

\section{CONCLUSION}

This paper presents a novel active charge balancing scheme that effectively removes the residual charge on an electrode by precisely controlling either the pulse width of current stimulus. The range of adjustment of pulse width should also be carefully selected to prevent altering neural excitability. This scheme is simulated with Matlab/Simulink and has shown to be effective for all mismatches tested, which included mismatches larger than those normally experienced with IC chips. Furthermore, the scheme is robust enough to perform well for an application such as the retinal prosthesis design where the amplitude of the stimulation pulses is variable based on the sensory input. The simulations also provide promising results that the charge balancing scheme will still be effective even using a comparator to monitor the residual electrode voltage, instead of a higher footprint high-bit ADC. A circuit realization of the scheme into our retina IC [5] is undergoing. Future goals include testing this prototype chip with electrodes in saline solution

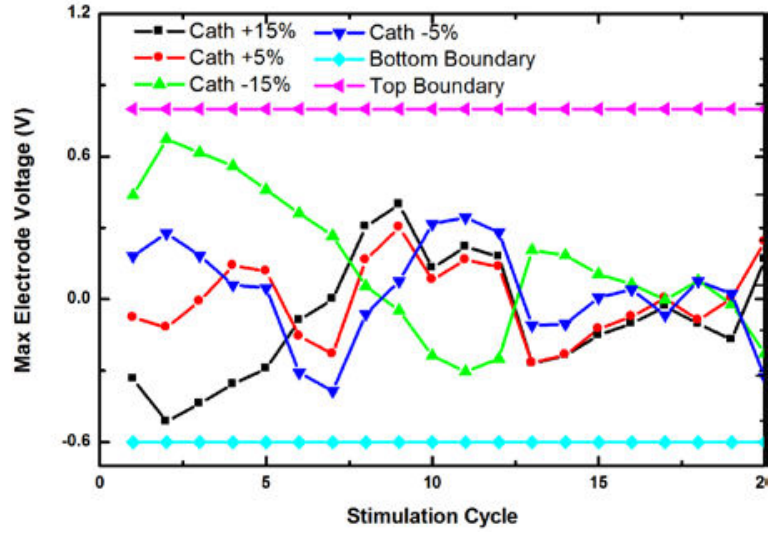

Fig. 6. The max electrode voltage during post-stimulation time with proposed control scheme with a 1-bit ADC (comparator) as feedback sensor and variable stimulation amplitudes. A larger ripple is observed because the comparator can only provide 1-bit information to the PID controller.

and also characterization of the feedback loop for improved settling time and stability. Detail of the hardware implementation will be discussed in our later publication.

\section{ACKNOWLEDGMENT}

This work is partially funded by NSF through BMES-ERC, UC Laboratory Fees Research Program, and the Endowment award for the Chan Soon-Shiong Bionic Engineering Research Center by California Capital Equity LLC.

\section{REFERENCES}

[1] D. R. Merrill, M. Bikson, and J. G. R. Jefferys, "Electrical stimulation of excitable tissue: design of efficacious and safe protocols," Journal of Neuroscience Methods, vol. 141, pp. 171-198, 2005

[2] D. Merrill, "The Electrochemistry of Charge Injection at the Electrode/Tissue Interface," in Implantable Neural Prostheses 2, D. Zhou and E. Greenbaum, Eds., ed: Springer New York, 2010, pp. 85138.

[3] S. Ji-Jon and R. Sarpeshkar, "A Low-Power Blocking-Capacitor-Free Charge-Balanced Electrode-Stimulator Chip With Less Than 6 nA DC Error for 1-mA Full-Scale Stimulation," Biomedical Circuits and Systems, IEEE Transactions on, vol. 1, pp. 172-183, 2007.

[4] H. Chun, Y. Yang, and T. Lehmann, "Safety Ensuring Retinal Prosthesis With Precise Charge Balance and Low Power Consumption," Biomedical Circuits and Systems, IEEE Transactions on, vol. PP, pp. 1-1, 2013.

[5] C. Kuanfu, L. Yi-Kai, and L. Wentai, "A 37.6mm<sup $>2</$ sup $>1024-$ channel high-compliance-voltage SoC for epiretinal prostheses," in Solid-State Circuits Conference Digest of Technical Papers (ISSCC), 2013 IEEE International, 2013, pp. 294-295.

[6]E. Noorsal, K. Sooksood, X. Hongcheng, R. Hornig, J. Becker, and M. Ortmanns, "A Neural Stimulator Frontend With High-Voltage Compliance and Programmable Pulse Shape for Epiretinal Implants," Solid-State Circuits, IEEE Journal of, vol. 47, pp. 244-256, 2012.

[7] K. Sooksood, T. Stieglitz, and M. Ortmanns, "An Active Approach for Charge Balancing in Functional Electrical Stimulation," Biomedical Circuits and Systems, IEEE Transactions on, vol. 4, pp. 162-170, 2010.

[8] G. Song and L. Hoi, "Biphasic-current-pulse self-calibration techniques for monopolar current stimulation," in Biomedical Circuits and Systems Conference, 2009. BioCAS 2009. IEEE, 2009, pp. 61-64.

[9] A. S. McCormack and K. R. Godfrey, "Rule-based autotuning based on frequency domain identification," Control Systems Technology, IEEE Transactions on, vol. 6, pp. 43-61, 1998.

[10] S. Shah, A. Hines, D. Zhou, R. J. Greenberg, M. S. Humayun, and J. D. Weiland, "Electrical properties of retinal-electrode interface," Journal of Neural Engineering, vol. 4, pp. S24-S29, Mar 2007. 